\title{
Prognostic value of p53 protein and MK-1 (a tumor-associated antigen) expression in gastric carcinoma
}

\author{
M. Salih Deveci and Güzin Deveci \\ Department of Pathology, Gülhane Military Medical Academy, Etlik 06018, Ankara, Turkey
}

\begin{abstract}
Background. MK-1, the target molecule of FU-MK-1, is encoded by the GA733-2 gene, which is currently being used as a target in clinical trials for gastric, intestinal and biliary cancer treatment with monoclonal antibodies. Also of interest is $\mathbf{p 3 3}$, a protein that has been intensively investigated in relation to particular types of tumors, patterns of metastases, tumor stage, and prognosis.

Methods. The expression of p53 protein and MK-1 antigen was investigated in specimens from 42 patients with gastric carcinoma. The specimens were stained by the avidin-biotin peroxidase technique for immunohistochemical examination.

Results. MK-1 was positive in $21(50 \%)$ of the 42 cases. MK-1 expression was more frequent in cardia tumors $(\mathbf{7 1} \%)$, in large $(>3 \mathrm{~cm})$ tumors $(60 \%-64 \%)$, and in specimens from patients with more than five metastatic lymph nodes $(69 \%)$. p53 expression was present in $20(48 \%)$ of the 42 cases. Of these 20 patients, $15(52 \%)$ had tubular adenocarcinoma (TA) and $5(38 \%)$ had signet ring cell carcinoma. p53 expression was more frequent in the tumors of male patients $(55 \%$ vs $27 \%)$; in poorly differentiated TAs (60\% vs $47 \%$ in well-tomoderately differentiated TAs); in smaller tumors $(\leq \mathbf{3} \mathrm{cm}$, $\mathbf{7 2} \%$ vs $\mathbf{4 3} \%-\mathbf{5 0} \%$ in larger tumors); in patients with a prominent inflammatory response $(61 \%$ vs $21 \% ; P<0.02)$; and in patients with lymphatic vessel invasion $(77 \%$ vs $34 \%$; $P<$ 0.02). However, p53 expression was less frequent in the presence of more than five metastatic lymph nodes $(23 \%$ vs $60 \%$ for five or fewer nodes; $P<\mathbf{0 . 0 5})$. Most patients with p53- and MK-1-positive gastric carcinomas and those more than five metastatic lymph nodes had a poor prognosis.

Conclusion. The study found that the expression of both p53 and MK-1 was frequent in aggressive gastric carcinomas; however, extensive lymph node involvement (more than five nodes) was the only significant factor related to overall survival.
\end{abstract}

Key words Gastric carcinoma $\cdot$ p53 protein · MK-1 antigen · Clinicopathological parameters $\cdot$ Prognosis

Offprint requests to: M.S. Deveci

Received: September 14, 2006 / Accepted: April 6, 2007

\section{Introduction}

Gastric carcinoma is one of the most common visceral cancers. The survival rate in patients with gastric carcinomas is rather low, even in developed countries. Therefore, besides the variables known to be prognostic markers, new independent parameters are being investigated [1]. To date, many tumor-associated antigens, either intracellular or on the cell surface, have been identified [2]. The $p 53$ gene product is the best known marker. In the past decade, it has been intensively investigated whether $p 53$ mutations are associated with particular types of tumors, patterns of metastases, and tumor stage or prognosis [1,3]. In gastric carcinomas, the frequency of tumors with p53 expression has been reported to vary from $4 \%$ to $71 \%$ [4-12].

MK-1, a tumor-associated antigen and glycoprotein marker on the tumor cell surface, reported by Watanabe et al. [13], is different from carcinoembryonic antigen (CEA), epithelial membrane antigen (EMA), keratin polypeptides, and other gastric carcinoma antigens, including sialylated CD1S (CSLEX1). Watanabe et al. [13] obtained it from the carcinoma cells of a poorly differentiated gastric adenocarcinoma. Tomita et al. [14] found that MK-1, the target molecule of FUMK-1, is encoded by the GA733-2 gene, which is currently being used as a target in clinical trials with monoclonal antibodies.

In the present study, p53 protein and MK-1 antigen expression was investigated in 42 patients with gastric carcinoma. The aim of the study was to show the presence of any relationship between pathological prognostic factors and the expressions of the monoclonal antibodies tested.

Patients, materials, and methods

This study was performed in 42 patients with primary gastric carcinoma. Demographic data of the patients 
were retrieved from the intrahospital information system of Gülhane Military Medical Academy, Turkey. All patients underwent preoperative diagnosis, resection, and postoperative follow-up at the same hospital. Thirty-one patients were male, 11 female. The mean age was 57.4 years (range, 27-84 years).

Formalin-fixed, paraffin-embedded tissue samples were obtained from the files of the Department of Pathology. Four-to-six-micron-thick sections were prepared from these tissues. Gastric carcinomas were classified as tubular adenocarcinoma and signet ring cell carcinoma according to the WHO system [15].

FU-MK-1 monoclonal antibody (MoAb) was provided by the Department of Pathology, Fukuoka University, Fukuoka, Japan. For p53 MoAb, a mouse antihuman, BP53-12, clone DO-7 (BioGenex Laboratories, San Ramon, CA, USA) was used. Immunohistochemically, the avidin-biotin peroxidase complex technique was employed. 3-amino-9-ethyl-carbazole (AEC) was used as the chromogen and Mayer's hematoxylin was used for counterstaining. Negative controls were prepared by replacing the primary antibodies with normal mouse serum.

The p53- and MK-1-positive cases were semiquantitatively categorized. Staining over $10 \%$ of tumor cells was accepted as positive expression.

Statistical analysis of clinicopathological parameters was done with the $\chi^{2}$ test and Fishers' exact test, using a significance level of 0.05 . The survival rates of the patients were estimated by the Kaplan-Meier method for p53- and MK-1-positive and -negative cases, and Cox regression analysis was used for all prognostic parameters. The SPSS 12.0 program (Chicago, IL, USA) for Windows was used for statistical analyses.

\section{Results}

MK-1 expression was detected in 21 gastric carcinoma cases. The expression was located in membrane or cytoplasm (Fig. 1A,B). p53 expression was observed in 20 gastric carcinoma cases. Immunoreaction of p53 was always nuclear (Fig. 2A,B). No staining with p53 or FUMK-1 was found in negative control slides.

p53 protein and MK-1 antigen expressions were examined in relation to the clinicopathological characteristics (Table 1). The frequency of p53 expression was comparatively higher $(55 \%)$ in male patients. Five $(38 \%)$ of 13 signet ring cell carcinomas (SRCCs) and 15 $(52 \%)$ of the 29 cases of tubular adenocarcinomas (TAs) showed p53 expression. In the poorly differentiated TA (PDTA) carcinomas, p53 expression was slightly higher than that in the well-differentiated to moderately differentiated (WD/MD) TAs (60\% vs $47 \%$, respectively). Tumors invading the serosa showed p53 expression

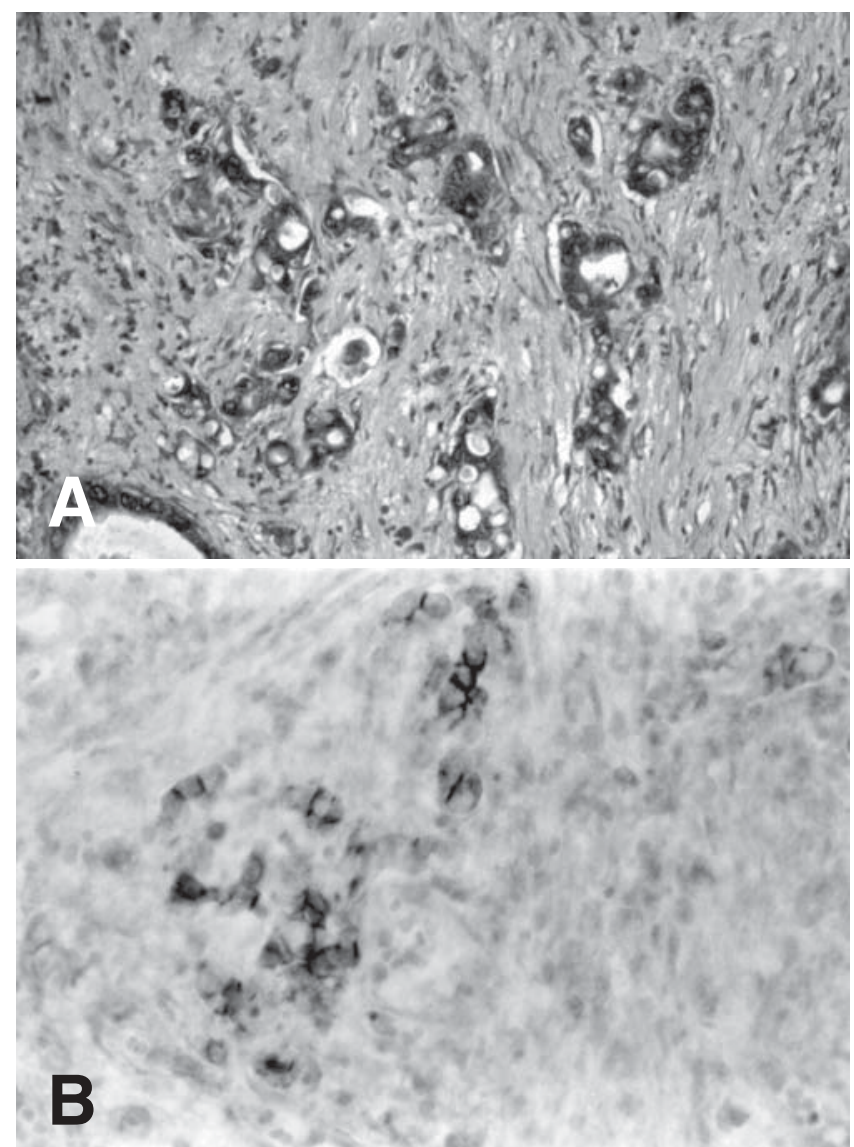

Fig. 1. MK-1 expression in gastric tubular adenocarcinoma (A) and in gastric signet ring cell carcinoma $(\mathbf{B})$. $\mathbf{A} \times 100$; B $\times 200$

similar to that in tumors confined to the muscularis propria. The p53 expression in patients without lymph node metastasis was higher than that in patients with lymph node metastasis (58\% vs 39\%). In addition, in patients with more than five metastatic lymph nodes, p53 expression was lower than that in patients with five or fewer metastatic lymph nodes $(23 \%$ vs $60 \% ; P<$ $0.05)$. Ten of the 13 gastric carcinoma with lymphatic vessel invasion $(77 \%)$ were positive for p53 $(P<0.02)$. p53 expression was higher in patients with a prominent lymphocytic response with many lymphoid follicles inside and/or surrounding the tumor (61\% vs $21 \%$; $P<0.02)$.

In relation to tumor site, MK-1 expression was highest $(71 \%)$ in tumors of the cardia; in relation to tumor size, MK-1 expression was lower in tumors of $3 \mathrm{~cm}$ or less ( $28 \%$ vs $60 \%-64 \%$ in larger tumors). MK-1 expression was positive in 5 of the SRCCs and in 16 of the TAs. MK-1 expression was higher in PDTA than in WD/MDTA carcinomas (70\% vs $47 \%$ ) and it was also higher in the presence of lymph node metastases than in lymph-node-negative patients. MK-1 expression was higher in patients with more than five metastatic lymph 


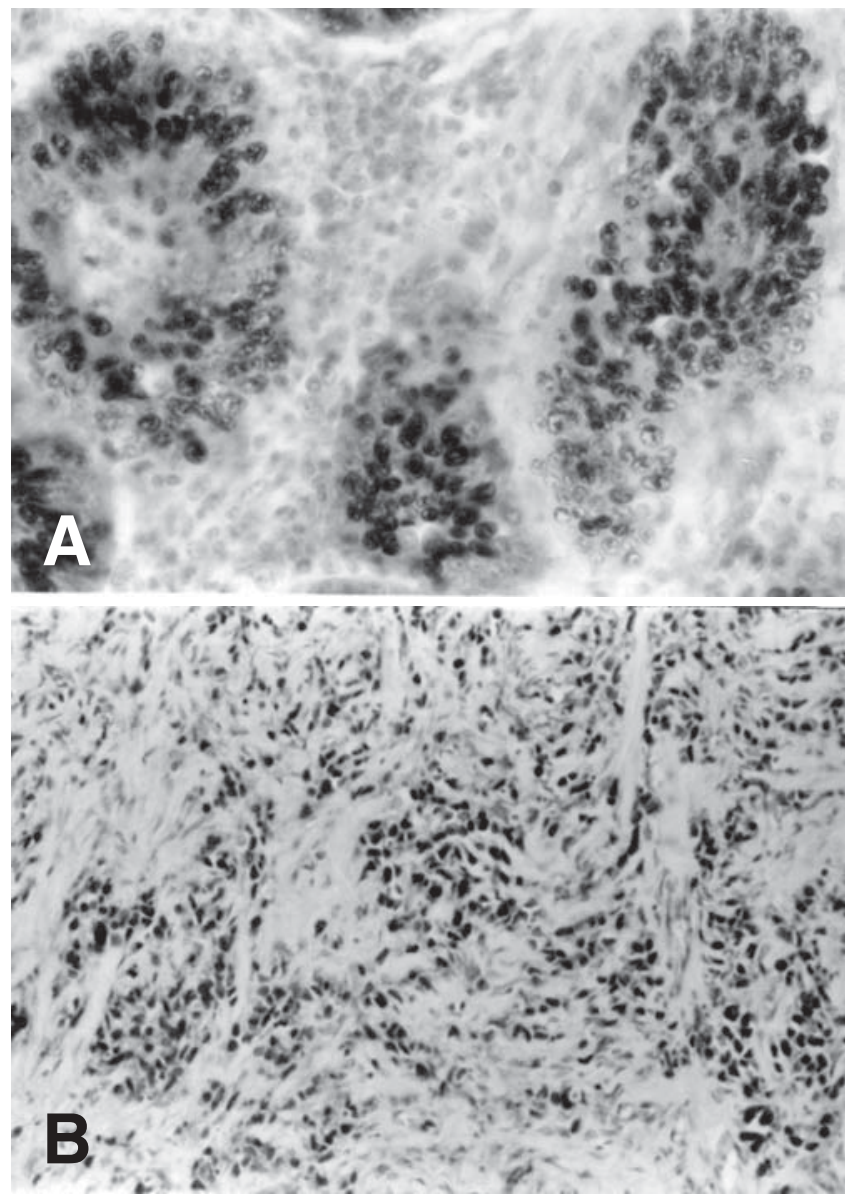

Fig. 2. Strong p53 expression in gastric tubular adenocarcinoma (A) and in gastric signet ring cell carcinoma (B). $\mathbf{A} \times 200 ; \mathbf{B} \times 100$

nodes than in those with five or fewer metastatic lymph nodes $(69 \%$ vs $40 \%)$.

Two of the 42 patients had early gastric carcinomas and both showed p53 expression while one of the two also showed positivity for MK-1. One of the 42 gastric carcinomas had metastasized to the liver and the other to the ovary. The primary tumors in these patients showed positivity for MK-1, but not for $\mathrm{p} 53$.

The mean follow-up time in the 42 patients with gastric carcinomas was 36 months (range, 5-65 months). Most patients with p53- or MK-1-positive gastric carcinomas generally showed a poor prognosis. However, survival analyses by the Kaplan-Meier method and Cox regression analysis revealed that the presence of extensive lymph node metastases (five or more) was the only significant factor related to overall survival.

\section{Discussion}

Carcinoembryonic antigen (CEA) is a useful marker for monitoring patients with gastric cancer and is widely used in immunohistological studies [16,17]. This molecule is known to have complex antigens consisting of a number of related antigens. Several investigators have recently reported MoAbs generated against gastrointestinal cancer antigens such as tumorassociated glycoprotein-72 (TAG-72) and stomach adenocarcinoma-associated antigen (CSTO-1) $[16,18]$.

Cytoplasmic expression of MK-1 is an important tumor marker for gastrointestinal system carcinomas, particularly those with a glandular pattern [13]. MK-1 may be used in the diagnosis - as well as in the differential diagnosis - of certain gastrointestinal carcinomas, such as metastatic gastric carcinoma, cholangiocarcinoma, and hepatocellular carcinoma [13].

The frequency of MK-1 immunoreactivity in the series reported by Watanabe et al. [13] was higher than that in the current study ( $85 \%$ vs $50 \%$ ). In our study, no relationship was found between MK-1 expression, age, sex, the histological type of carcinoma, or depth of invasion. However, tumors $3 \mathrm{~cm}$ or less in size showed lower MK-1 expression than larger tumors (28\% vs $60 \%-64 \%$ ). MK-1 expression was higher in tumors of the cardia than in tumors at other sites, and it was higher in large $(>3 \mathrm{~cm})$ tumors; MK-1 expression was also higher in the presence of more than five lymph node metastases than in the presence of fewer lymph node metastases. Hamada et al. [19] documented that MK-1 expression was an independent prognostic marker of increased survival in carcinomas of the ampulla of Vater. However, MK-1 expression in our series was associated with late-stage survival, but not overall survival.

Ueno et al. [20] have recently reported a genetically engineered fusion protein of the superantigen staphylococcal enterotoxin A (SEA) and anti-MK-1 scFv antibody $[\mathrm{SEA} / \mathrm{FUscFv}$ (single chain fragment antibody derived from FU-MK-1)], a potentially useful T-cell immunotherapeutic reagent for human MK-1-expressing tumors. In vivo administration of $\mathrm{FUscFv} / \mathrm{IL}-2$ fusion protein induces the localization of interleukin (IL)-2 to tumor tissues, as reported by Matsumoto et al. [21] Locally increased IL-2 supports the immune response to human MK-1-expressing tumors while reducing the systemic side-effects of IL-2. The clinical benefit of the in vivo administration of anti-MK- $1 \mathrm{scFv}$ antibody in human MK-1-expressing gastric carcinomas is not known yet. In our series, half of the MK-1-positive gastric carcinomas were associated with a prominent lymphocyterich inflammatory response. Anti-MK-1 scFv antibody may trigger local IL-2 release from the lymphocytic component and play an important role in inhibiting MK-1-expressing gastric carcinoma cells.

The $p 53$ gene is known to play an important role in the regulation of cell proliferation. It has been suggested that loss of normal $p 53$ function is associated 
Table 1. Expression of p53 protein and MK-1 antigen and clinicopathological parameters in 42 patients with gastric carcinoma

\begin{tabular}{|c|c|c|c|}
\hline & No. & p53 (+) & $\mathrm{MK}-1(+)$ \\
\hline Total & 42 & $20(48 \%)$ & $21(50 \%)$ \\
\hline \multicolumn{4}{|l|}{ Sex } \\
\hline Male & 31 & $17(55 \%)$ & $16(52 \%)$ \\
\hline Female & 11 & $3(27 \%)$ & $5(45 \%)$ \\
\hline \multicolumn{4}{|l|}{ Age (years; mean, 57.4) } \\
\hline$\leq 55$ & 17 & $8(47 \%)$ & $8(47 \%)$ \\
\hline$>55$ & 25 & $12(48 \%)$ & $13(52 \%)$ \\
\hline \multicolumn{4}{|l|}{ Site of tumor } \\
\hline Antrum & 24 & $13(54 \%)$ & $11(46 \%)$ \\
\hline Corpus & 7 & $3(43 \%)$ & $2(29 \%)$ \\
\hline Cardia & 7 & $3(43 \%)$ & $5(71 \%)$ \\
\hline Linitis plastica & 4 & $1(25 \%)$ & $1(25 \%)$ \\
\hline \multicolumn{4}{|l|}{ Tumor size (cm; mean, 4.5) } \\
\hline$\leq 3$ & 18 & $13(72 \%)$ & $5(28 \%)$ \\
\hline $3-6$ & 14 & $6(43 \%)$ & $9(64 \%)$ \\
\hline$>6$ & 10 & $5(50 \%)$ & $6(60 \%)$ \\
\hline \multicolumn{4}{|l|}{ Pattern } \\
\hline Signet ring cell carcinoma & 13 & $5(38 \%)$ & $5(38 \%)$ \\
\hline Tubular adenocarcinoma (TA) & 29 & $15(52 \%)$ & $16(55 \%)$ \\
\hline \multicolumn{4}{|l|}{ Differentiation (TA) } \\
\hline Well-to-Moderate & 19 & $9(47 \%)$ & $9(47 \%)$ \\
\hline Poor & 10 & $6(60 \%)$ & $7(70 \%)$ \\
\hline \multicolumn{4}{|l|}{ Depth of invasion } \\
\hline Mucosa and submucosa & 3 & $2(67 \%)$ & $1(33 \%)$ \\
\hline Muscularis propria & 6 & $2(33 \%)$ & $3(50 \%)$ \\
\hline Serosa & 33 & $16(48 \%)$ & $17(52 \%)$ \\
\hline \multicolumn{4}{|l|}{ Lymph node metastasis } \\
\hline Negative & 19 & $11(58 \%)$ & $8(42 \%)$ \\
\hline Positive & 23 & $9(39 \%)$ & $13(56 \%)$ \\
\hline$\leq 5$ & 10 & $6(60 \%) *$ & $4(40 \%)$ \\
\hline$>5$ & 13 & $3(23 \%)$ & $9(69 \%)$ \\
\hline \multicolumn{4}{|l|}{ Lymphatic vessel invasion } \\
\hline$(-)$ & 29 & $10(34 \%)$ & $16(55 \%)$ \\
\hline$(+)$ & 13 & $10(77 \%)^{* *}$ & $5(38 \%)$ \\
\hline \multicolumn{4}{|l|}{ Inflammatory response } \\
\hline$(-)$ & 14 & $3(21 \%) * *$ & $7(50 \%)$ \\
\hline$(+)$ & 28 & $17(61 \%)$ & $14(50 \%)$ \\
\hline
\end{tabular}

with cell transformation and the development of neoplasms [1,3]. Abnormalities of the $p 53$ gene have been demonstrated in a wide variety of common human malignancies $[1,3]$.

In gastric carcinomas, the frequency of $\mathrm{p} 53$ expression varies from $4 \%$ to $71 \%$ (mean, $50 \%$ ) [4-12]. Martin et al. [22] detected p53 expression in $57 \%$ of gastric carcinoma cases. Fukunaga et al. [7] reported p53 positivity in $49 \%$ of gastric carcinoma cases. They detected significantly higher p53 positivity in intestinal-type carcinomas than in diffuse-type carcinomas ( $56 \%$ vs $27 \%$ ). Hurlimann and Saraga [4] showed that $11 \%$ of gastric carcinomas of the diffuse type were positive for p53 compared with $71 \%$ of carcinomas of the intestinal type. In the present study, no relationship was found between $\mathrm{p} 53$ expression and age, tumor site, or histological type. In our study, sig- net ring cells showed p53 immunoreactivity, which was also reported by Fukunaga et al. [7]. These cells may have originated from nonintestinal goblet cells [23]. One possible determinant of the different frequencies of p53 immunoreactivity in various types of gastric carcinomas may be aneuploidy [24]. Tamura et al. [8] support this observation, reporting p53 expression in $64 \%$ of aneuploid gastric carcinomas.

The p53 mutation is accepted as an early event in gastric carcinomas $[1,4,24]$. A correlation between $\mathrm{p} 53$ expression and the presence of metastases was noted by Kakeji et al. [25], and Starzynska et al. [6] However, Hurlimann and Saraga [4], Fukunaga et al. [7], and Gabbert et al. [9] have shown no relationship of p53 expression with liver metastasis or lymph node involvement. In our series, the presence of p53 expression in 
early carcinomas, in tumors of $3 \mathrm{~cm}$ or less, and in patients with a lymphocytic response, as well as the absence of a relationship between p53 expression and lymph node metastases, suggest that $\mathrm{p} 53$ positivity could favor an early event in gastric carcinomas.

We found lower p53 expression in patients with metastases in more than five lymph nodes compared with p53 expression in those with five or fewer nodes. However, there was higher p53 expression in patients with lymphatic vessel invasion regardless of lymph node metastasis. This feature may be related to early hematogenous metastasis in gastric carcinomas as an independent prognostic factor. Schneider et al. [11] and Gabbert et al. [9] have reported observations in accordance with ours in this regard. However, on the contrary, Kakeji et al. [25] reported that there was a possible relationship between p53 expression and lymph node metastasis.

In conclusion, the results of the present study showed that MK-1 antigen was correlated to late pathological stage in gastric carcinomas. p53 was shown to be a relatively early marker for tumor progression from well- to poorly differentiated tumors and was related to lymphatic vessel invasion regardless of lymph node metastasis. Both $\mathrm{p} 53$ protein and MK-1 antigen were shown to be associated with the aggressive features of gastric cancers. However, in this series, overall survival was related only to extensive lymph node (more than five) metastesis.

Acknowledgments We thank Dr. Hiroshi Iwasaki (M.D., Professor), of the Pathology Department of Fukuoka University (Japan), for his supply of the FUMK-1 MoAb used in this study.

\section{References}

1. Chang F, Syrjänen S, Kurvinen K, Syrjänen K. The p53 tumor suppressor gene as a common cellular target in human carcinogenesis. Am J Gastroenterol 1993;88:174-86.

2. Campbell FA, Redmond HP, Bouchier-Hayes D. The role of tumor rejection antigens in host antitumor defense mechanisms. Cancer 1995;75:2649-55.

3. Chang F, Syrjänen S, Tervahauta A, Syrjänen K. Tumourigenesis associated with the p53 tumour suppressor gene. Br J Cancer 1993;68:653-61.

4. Hurlimann J, Saraga EP. Expression of p53 protein in gastric carcinomas. Association with histologic type and prognosis. Am J Surg Pathol 1994;18:1247-53.

5. Sasano H, Date F, Imatani A, Asaki S, Nagura H. Double immunostaining for c-erbB-2 and p 53 in human stomach cancer cells. Hum Pathol 1993;24:584-9.

6. Starzynska T, Bromley M, Ghosh A, Stern PL. Prognostic significance of p53 overexpression in gastric and colorectal carcinoma. Br J Cancer 1992;66:558-62.

7. Fukunaga M, Monden T, Nakanishi H, Ohue M, Fukuda K, Tomita N, et al. Immunohistochemical study of p53 in gastric carcinoma. Am J Clin Pathol 1994;101:177-80.
8. Tamura G, Kihana T, Nomura K, Terada M, Sugimura T, Hirohashi S, et al. Detection of frequent p53 gene mutations in primary gastric cancer by cell sorting and polymerase chain reaction single-strand conformation polymorphism analysis. Cancer Res 1991;51:3056-8.

9. Gabbert HE, Müller W, Schneiders A, Meier S, Hommel G. The relationship of p53 expression to the prognosis of 418 patients with gastric carcinoma. Cancer 1995;76:720-6.

10. Joypaul BV, Hopwood D, Newman EL, Qureshi S, Grant A, Ogston SA, et al. The prognostic significance of the accumulation of p53 tumour suppressor gene protein in gastric adenocarcinoma. Br J Cancer 1994;69:943-6.

11. Schneider BG, Hilsenbeck SG, Hensel CH, Pekkel V, Shelton $\mathrm{CH}$, Rodriguez-Martinez HA, et al. p53 mutations in gastric and colorectal cancers in Texas Hispanics versus Anglos. Virchows Arch 1994:424:187-93.

12. Pinto-de-Sousa J, Silva F, David L, Leitao D, Seixas M, Pimenta A, Cardoso-de-Oliveira M. Clinicopathological significance and survival influence of $\mathrm{p} 53$ protein expression in gastric carcinoma. Histopathology 2004;44:323-31.

13. Watanabe R, Johzaki H, Iwasaki H, Kikuchi M, Ikeda S. A new tumor-associated antigen defined by a monoclonal antibody directed to gastric adenocarcinoma. Cancer 1993;71:243947.

14. Tomita Y, Arakawa F, Yamamoto T, Kuwahara M, Watanabe R, Iwasaki $\mathrm{H}$, et al. Molecular identification of a human carcinomaassociated glycoprotein antigen recognized by mouse monoclonal antibody FU-MK-1. Jpn J Cancer Res 2000;91:231-8.

15. Hamilton SR, Aaltonen LA. Pathology and genetics of tumors of the digestive system. Lyon: IARC; 2000. p. 37-52.

16. Guadagni F, Roselli M, Amato T, Cosimelli M, Mannella E, Perri $\mathrm{P}$, et al. Tumor-associated glycoprotein-72 serum levels complement carcinoembryonic antigen levels in monitoring patients with gastrointestinal carcinoma. Cancer 1991;68:2443-50.

17. Pascal RR, Mesa-Tejada R, Bennett SJ, Garces A, Fenoglio M. Carcinoembryonic antigen. Immunohistologic identification in invasive and intraepithelial carcinomas of lung. Arch Pathol Lab Med 1977;101:568-71.

18. Togashi H, Terasaki PI, Chia D, Kukes G, Mugishima H, Kasai $\mathrm{K}$, et al. A monoclonal antibody, CSTO-1, against a stomach adenocarcinoma-associated antigen. Cancer Res 1984;44:39526.

19. Hamada Y, Nakayama Y, Mizoguchi M, Ikeda S, Kuroki M, Iwasaki H. MK-1 expression in carcinoma of the ampulla of Vater as a predictor of improved prognosis after surgical resection. Cancer Lett 2006;7:1-6.

20. Ueno A, Arakawa F, Abe H, Matsumoto H, Kudo T, Asano R, et al. T-cell immunotherapy for human MK-1-expressing tumors using a fusion protein of the superantigen SEA and anti-MK-1 scFv antibody. Anticancer Res 2002;22:769-76.

21. Matsumoto H, Liao S, Arakawa F, Ueno A, Abe H, Awasthi A, et al. Targeting of interleukin-2 to human MK-1-expressing carcinoma by fusion with a single-chain Fv of anti-MK-1 antibody. Anticancer Res 2002;22:2001-7.

22. Martin HM, Filipe MI, Morris R. P53 expression and prognosis in gastric carcinoma. Int J Cancer 1992;50:859-62.

23. Tatematsu M, Furihata C, Katsuyama T, Miki K, Honda H, Konishi Y, et al. Gastric and intestinal phenotypic expressions of human signet ring cell carcinomas revealed by their biochemistry, mucin histochemistry, and ultrastructure. Cancer Res 1986;46: 4866-72.

24. Auer GU, Heselmeyer KM, Steinbeck RG, Munck-Wikland E, Zetterberg AD. The relationship between aneuploidy and p53 overexpression during genesis of colorectal adenocarcinoma. Virchows Arch 1994;424:343-7.

25. Kakeji Y, Korenaga D, Tsujitani S, Baba H, Anai H, Maehara Y, et al. Gastric cancer with p53 overexpression has high potential for metastasizing to lymph nodes. Br J Cancer 1993;67:589-93. 